\title{
Factores de impacto en el aprendizaje virtual en los estudiantes de la Universidad Católica Boliviana "San Pablo" Regional Cochabamba
}

\section{Impact factors of e-learning in students of Universidad Católica Boliviana San Pablo Regional Cochabamba}

\author{
Samuel Israel Goyzueta Rivera \\ sgoyzuetar@univalle.edu \\ Código ORCID: 0000-0003-0965-2235 \\ Universidad Privada del Valle, Tiquipaya, Bolivia
}

- Artículo recibido en octubre 2020

- Arbitrado en noviembre 2020

- Aceptado en diciembre 2020

RESUMEN

- Publicado en enero 2021

\begin{abstract}
El presente estudio tuvo como objetivo general identificar los factores de mayor impacto en el aprendizaje virtual en los estudiantes de la Universidad Católica Boliviana "San Pablo", Regional Cochabamba (UCBSP-CBA). Para lograr este objetivo, se analizó la relación entre los constructos teóricos del Modelo de aceptación de la tecnología de Davis (1985) y la Teoría del comportamiento planeado de Ajzen (1991). Se encuestó a un total de 742 estudiantes de pregrado de la UCBSP-CBA. Los resultados de esta encuesta se analizaron mediante la técnica de ecuaciones estructurales PLS-SEM, que ayudó a dar respuesta a las hipótesis planteadas. Los resultados demostraron que el control conductual, la utilidad percibida y la norma subjetiva tienen impacto positivo en la adaptación del aprendizaje virtual, pero la actitud tiene un impacto negativo en la misma.
\end{abstract}

Palabras clave: Aprendizaje virtual; educación superior; ecuaciones estructurales; PLS

\section{ABSTRACT}

The general purpose of this study is to identify the factors that have the main impact on elearning in students at Universidad Católica Boliviana San Pablo, Regional Cochabamba (UCBS-CBA). To achieve this goal, it was analyzed the relationship between the theoretical constructs of Davis' Technology acceptance model (1985) and Ajzen's Theory of planned behavior (1991). It was surveyed a total of 742 undergraduate students of UCBSP-CBA; the results of this survey were analyzed by the structural equation technique PLS-SEM, which helped to give answer the hypotheses stated. The results showed that behavioral control, perceived utility and subjective norm have a positive impact on e-learning adoption, while attitude has a negative impact on it.

Keywords: E-learning; higher education; structural equation modelling; PLS 


\section{INTRODUCCIÓN}

El aprendizaje virtual juega un papel importante en la formación de los estudiantes, más aun en estos tiempos cuando las instituciones se vieron en la necesidad de cerrar sus puertas con el fin de prevenir y evitar contagios.

El sistema de enseñanza tradicional durante mucho tiempo se ha dado en un salón de clases con un profesor dando discursos a los estudiantes y ellos escuchando y tomando notas, en este sistema comunicación entre ambos actores ha sido identificada como un componente importante de aprendizaje.

Sin embargo, las novedades $\mathrm{y}$ avances en los sistemas de enseñanza han desafiado las actitudes tradicionales hacia la educación y sus métodos (Haverila, $\mathrm{M}$. y Barkhi, N., 2009; Tamrakar A. y Mehta, K., 2011). La tecnología incita la aplicación de nuevas herramientas con el objetivo de hacer este proceso más efectivo e interesante para los estudiantes.

Gracias a esto muchas universidades están empezando a implementar el aprendizaje virtual de manera regular. Los beneficios que brinda este tipo de educción son varios entre los cuales se destacan la eliminación de barreras, flexibilidad de tiempo y horarios entre otras (Haverila, M. y Barkhi, N,. 2009; Tamrakar A. y Mehta, K., 2011).

Existen algunas teorías como la del comportamiento planificado (TPB) y modelos específicos como el de la aceptación de la tecnología que se han utilizado como base para estudiar los elementos motivacionales y contextuales que influyen en la intención y adopción de estas actividades (Garavan, T., Carbery, R., O'Malley, G., y O'Donnell, D., 2010).

Debido a estos cambios hay una creciente necesidad de una entrega flexible de la educación, por lo que es importante conocer los factores de impacto en el aprendizaje virtual que permita a las universidades, y en caso en particular a la UCBSP-CBA, realizar y mejorar sus estrategias y modelo de educación.

\section{MÉTODO}

Las características asumidas en la investigación permiten definirla como un estudio de naturaleza cuantitativa, de carácter no experimental y de tipo 
transversal o transeccional, lineamientos que le confieren ciertas cualidades muy particulares al desarrollo del trabajo.

La investigación se realizó con base al enfoque cuantitativo, ya que mide la magnitud de los fenómenos y determina el grado de influencia de estos mediante un análisis estadístico.

Se utilizó el método deductivo debido a que se estudió una muestra determinada sin afectar los fenómenos observables, siguiendo un patrón estructurado para la recolección de información y posterior generalización de los resultados obtenidos, en continuidad con los estudios de Hernández, Fernández y Baptista (2014).

Este trabajo de investigación fue de carácter concluyente ya que se planteó como objetivo "probar hipótesis específicas y examinar relaciones particulares" (Malhotra, N., 2008, p.79).

La fuente de información utilizada para desarrollar la investigación fue a través de fuentes primarias. Se recolectó información de primera mano a través de encuestas a los estudiantes de diversas carreras de la UCBSP-CBA, por medio de un cuestionario como instrumento de recogida de datos. El periodo del levantamiento de la encuesta se lo realizó entre los meses de abril y mayo de la gestión 2020.

La población-meta del presente trabajo de investigación la conformaron estudiantes matriculados en las diferentes 17 carreras que oferta la UCBSP-CBA con una franja etaria entre los 17 años y 24 años de edad. El marco de muestreo es la representación de los elementos de la población-meta y consiste en un listado o conjunto de instrucciones para identificar ésta (Malhotra, N., 2008). En este caso, el marco muestral es la lista de los 3.479 estudiantes matriculados y activos para el primer semestre de la gestión 2020 de las 17 diferentes carreras ofertadas por la UBCSP-CBA. Se utilizó el muestreo no probabilístico por juicio que es una forma de muestreo por conveniencia (Malhotra, N., 2008); permite al investigador escoger la población de interés basándose en su experiencia o su juicio debido a que los considera representativos o que, de alguna manera, resultan adecuados. 
El cálculo de la muestra se obtuvo mediante el análisis de la potencia a priori, el cual se realiza antes de que se lleve a cabo un estudio que determina el tamaño de muestra apropiado para el objetivo. El cálculo se realizó con ayuda del software $G^{*}$ Power 3.1.9.7. Para determinar $\alpha$, se tomó un nivel de significancia de $1 \%$ y una potencia del 0.99. Para el tamaño del efecto Cohen, se establece estandarizadas del tamaño (Cohen, J., 1988); se propuso una categorización simple del tamaño del efecto pequeño (0.02), moderado (0.15) y grande (0.35). En este caso, se pudo inferir un tamaño de efecto moderado y, por último, el número de constructos del modelo que es 10. Aplicando los datos, se procedió al cálculo y se obtuvo un total de 279 personas a ser encuestadas. Sin embargo, en total se realizaron 742 encuestas mejorando la precisión estadística de los datos obtenidos.

\section{Modelos de adopción de tecnología}

Los avances tecnológicos siempre jugarán un rol vital en el campo de la administración. El progreso tecnológico alberga también la diseminación del conocimiento. Pero, hasta que este no sea utilizado o aceptado, la tecnología se convierte en algo innecesario (Oye, D., A. Iahad. y Ab.Rahim, N. Z., 2012). Dada la importancia de los avances tecnológicos a lo largo del tiempo, es necesario el análisis de su adopción evitando que estos sean considerados innecesarios, como exponen los autores.

El término Adopción se aplica a un nivel individual, la difusión puede ser entendida como la adopción por las masas. La adopción tecnológica conducirá a la difusión (Sharma, S., Hasteer, N., Mishra, S. P. y Van Belle, J. P., 2016). Más aún, el entendimiento respecto a la adopción tecnológica es de suma valía y, por ende, fundamental su definición.

Para Carr, la adopción tecnológica hace referencia a la etapa de selección de la tecnología para el uso de un individuo o una organización (Carr Jr, V. H., 1999).

El autor indica que la adopción toma en cuenta la elección de la herramienta tecnológica de una persona o empresa para su uso; no solo se enfoca en el uso del instrumento, sino en su análisis. 
Por otro lado, la adopción tecnológica puede ser definida como la voluntad dentro de un grupo de usuarios para utilizar la tecnología hacia su beneficio (Samaradiwakara, G. D. M. N. y Gunawardena, C. G., 2014).

Gracias a las definiciones anteriores podemos concluir que la adopción tecnológica está relacionada con la intención de seleccionar y utilizar herramientas tecnológicas con el objetivo de obtener algún beneficio, el cual puede darse en distintos ámbitos.

Muchos estudios han revelado que la adopción tecnológica no se relaciona con los aspectos tecnológicos por sí solos; empero ha ido evolucionando hacia un proceso mucho más complejo que involucra otras dimensiones: la actitud del usuario y su personalidad Venkatesh, V., 2000; Venkatesh, V., Thong J. Y., y Xu, X., 2012 ), influencia social (Ajzen, J. y Fishbein M. 1980), confianza (Gefen, Karahanna y Straub, 2003) y numerosas condiciones facilitadoras (Thompson, M. K., Moroni, G., Vaneker, T., Fadel, G., Campbell, R. I., Gibson, I. y Martina, F. , 2016). Entonces se puede inferir que los factores que influencian la aceptación de una determinada tecnología son muy variados y estudiados por diversas investigaciones $(\mathrm{Gu}$, Ja-Chul y Lee, Sang-Chul y Suh, Yung., 2009; Kim, Gi., Shin, Bongsik y Lee, Ho., 2009; Luarn, P. y Lin, H.H., 2005; Santos, D., Veiga, R. y Moura, L., 2010 y Venkatesh, V., 2000) que buscan proponer modelos aclaratorios de tal aceptación.

En la Tabla 1, se muestran los diversos modelos de aceptación de tecnología, con sus respectivos constructos, propuestos para analizar la aceptación de tecnología. 
Tabla 1. Modelos de Aceptación de Tecnología

\begin{tabular}{|c|c|c|c|}
\hline Sigla & Modelo & Constructos & Autor(es) \\
\hline TRA & $\begin{array}{l}\text { Teoría de acción } \\
\text { racional }\end{array}$ & $\begin{array}{l}\text { Normas subjetivas; Actitud } \\
\text { para el comportamiento }\end{array}$ & $\begin{array}{l}\text { Ajzen, J. y Fishbein } \\
\text { M. (1980) }\end{array}$ \\
\hline TAM & $\begin{array}{l}\text { Modelo de aceptación } \\
\text { de tecnología }\end{array}$ & $\begin{array}{l}\text { Normas subjetivas } \\
\text { Facilidad de uso percibida } \\
\text { Utilidad percibida }\end{array}$ & Davis (1985) \\
\hline MM & Modelo motivacional & $\begin{array}{l}\text { Motivación Intrínseca } \\
\text { Motivación Extrínseca }\end{array}$ & $\begin{array}{l}\text { Vallerand, R. J. (1997) } \\
\text { Davis F. D., Bagozzi, } \\
\text { R.P. y Warshaw, P.R } \\
\text { (1992) }\end{array}$ \\
\hline TPB & $\begin{array}{l}\text { Theory of planned } \\
\text { behavior }\end{array}$ & $\begin{array}{l}\text { Actitud para el } \\
\text { comportamiento Normas } \\
\text { subjetivas Control } \\
\text { comportamental percibido }\end{array}$ & Ajzen, I.(1985) \\
\hline TAM-TPB & $\begin{array}{l}\text { Modelo combinado } \\
\text { TAM-TPB }\end{array}$ & $\begin{array}{l}\text { Actitud para el } \\
\text { comportamiento } \\
\text { Normas subjetivas Control } \\
\text { comportamental percibido } \\
\text { Utilidad percibida }\end{array}$ & $\begin{array}{l}\text { Taylor, S. y Todd, P. } \\
\text { (1995) }\end{array}$ \\
\hline TRI & $\begin{array}{l}\text { Índice de disposición } \\
\text { a la tecnología }\end{array}$ & $\begin{array}{l}\text { Optimismo } \\
\text { Innovación Disconformidad } \\
\text { Inseguridad }\end{array}$ & $\begin{array}{l}\text { Parasuraman, A. } \\
(2000)\end{array}$ \\
\hline MPCU & $\begin{array}{l}\text { Modelo de utilización } \\
\text { de PC }\end{array}$ & $\begin{array}{l}\text { Ajuste del trabajo } \\
\text { Complejidad Consecuencias } \\
\text { a largo plazo } \\
\text { Efectos sobre la razón de } \\
\text { uso } \\
\text { Factores sociales } \\
\text { Condiciones facilitadoras }\end{array}$ & $\begin{array}{l}\text { Thompson, M. K., } \\
\text { Moroni, G., Vaneker, } \\
\text { T., Fadel, G., } \\
\text { Campbell, R. I., } \\
\text { Gibson, I. y Martina, } \\
\text { F. (2016) }\end{array}$ \\
\hline - & $\begin{array}{l}\text { Teoría de difusión de } \\
\text { la innovación }\end{array}$ & $\begin{array}{l}\text { Ventaja relativa Facilidad de } \\
\text { uso } \\
\text { Imagen } \\
\text { Visibilidad Compatibilidad } \\
\text { Demostración de resultados } \\
\text { Uso voluntario }\end{array}$ & $\begin{array}{l}\text { Rogers E. M. (1995) } \\
\text { Moore G. C. y } \\
\text { Benbasat I. (1996) }\end{array}$ \\
\hline- & $\begin{array}{l}\text { Teoría Social } \\
\text { Cognitiva }\end{array}$ & $\begin{array}{l}\text { Expectativas de los } \\
\text { resultados de desempeño y } \\
\text { personas Autoeficacia } \\
\text { Afecto } \\
\text { Ansiedad }\end{array}$ & $\begin{array}{l}\text { Compeau, D. R. y } \\
\text { Higgins, C. A. (1995). }\end{array}$ \\
\hline UTAUT & $\begin{array}{l}\text { Teoría unificada de } \\
\text { aceptación y uso de la } \\
\text { tecnología }\end{array}$ & $\begin{array}{l}\text { Expectativa de desempeño } \\
\text { Expectativa de esfuerzo } \\
\text { Influencia social } \\
\text { Condiciones facilitadoras }\end{array}$ & Venkatesh, V. (2000). \\
\hline
\end{tabular}

Fuente: Elaboración propia (2020) en base a Bobsin, D., Visentini, M. S. y Rech, I. (2009) 
Modelo de adopción de tecnología (TAM)

El modelo TAM propuesto por Davis (1985) examina el proceso de adopción de la tecnología a través de las percepciones de los usuarios finales en función de su utilidad y la facilidad de uso. El TAM considera variables como la utilidad percibida y la percepción de facilidad de uso. Usando el TAM, Davis (1993) se dio cuenta que la actitud del usuario determina la adopción de una tecnología en particular y el desarrollo de esta actitud se ve contribuida por la percepción de utilidad y la percepción de facilidad de uso. El poder predictivo del

TAM es una de sus principales fortalezas.
Este modelo ha sido verificado como un instrumento de predicción del uso de tecnología debido a su versatilidad y potencial de explicación y predicción, constituyéndose como uno de los más dominantes en la literatura en estas últimas décadas (Davis, F. D., 1985; Davis, 1993; Venkatesh, V. y Davis, F. D., 2000; Davis, F. D. y Venkatesh, V., 1996; Park, S., 2009). Dentro del ámbito tecnológico, ha sido aplicado en diferentes estudios: sitios web (Koufaris, M., 2002), correo electrónico (Szajna, B., 1996), cursos especializados en-línea (Hew, K. F. y Cheung, W. S., 2014), entre otros. La Figura 1 muestra el modelo original de TAM.

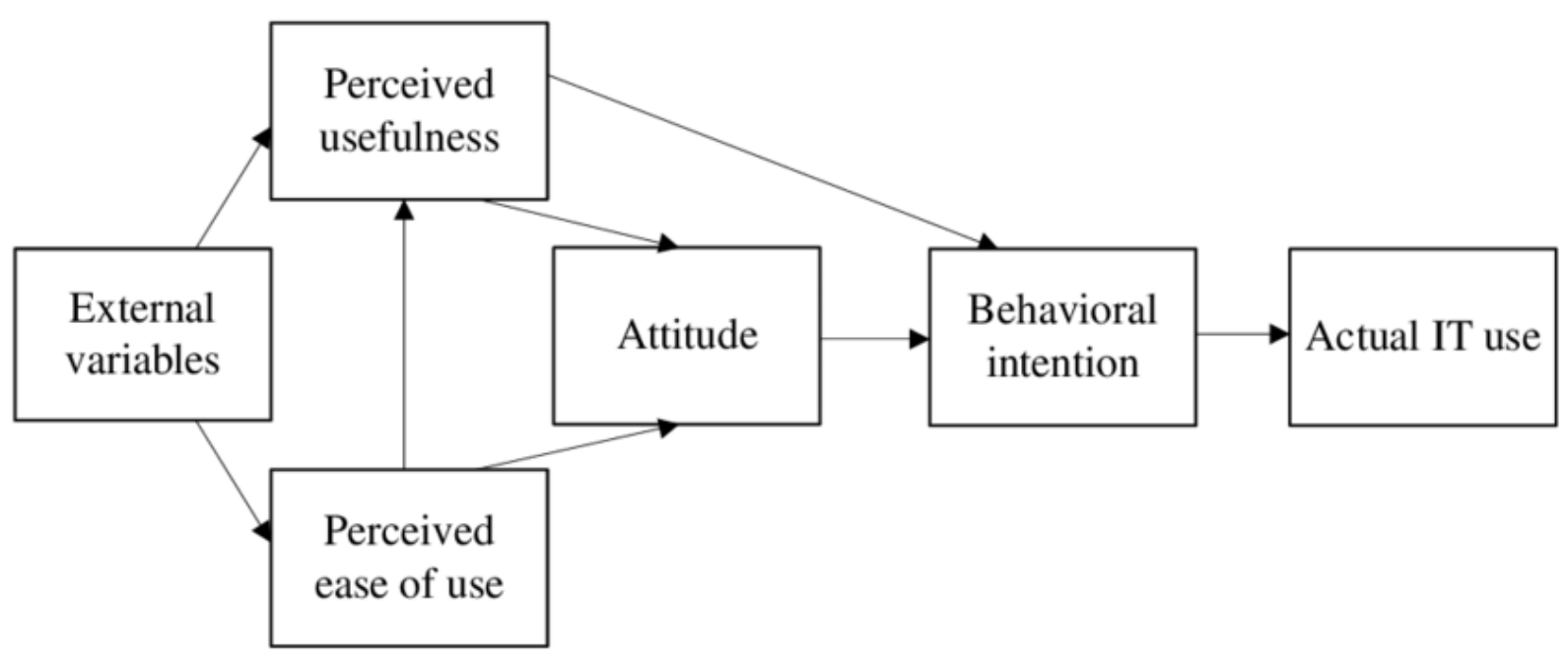

Figura 1. Modelo TAM. (Fuente: Davis, F. D., Bagozzi, R. P. y Warshaw, P.R., 1989) 


\section{Variables externas}

Las variables externas afectarán la intención conductual del individuo a través de la facilidad de uso percibida y la utilidad percibida; dependerán del contexto; pueden usarse para ampliar el modelo o abordar características únicas de la investigación (Musa, P. F., 2006). Para identificar variables externas se sugiere una revisión de literatura, ya que ayudará a elaborar fundamentos teóricos referentes al área de investigación. En un inicio, las primeras aplicaciones del modelo fueron en el área empresarial; por ese motivo, las variables externas contemplaban lo referente a ese entorno (Davis, F. D. y Venkatesh, V., 1996; Han, S., 2003). Actualmente y debido al amplio uso del modelo TAM, las variables externas pueden ser de diversa índole en mérito al tema de estudio.

\section{Utilidad percibida}

La utilidad percibida es definida como el grado en que una persona cree que el uso de una tecnología especifica pueda mejorar su rendimiento laboral (Chinyamurindi, W. y Shava, H., 2015). Por lo tanto, se puede entender la utilidad percibida como la medida en que uno cree que usar un sistema mejorará el rendimiento en un ámbito en específico. Varios estudios realizados concluyeron que la utilidad percibida es el principal determinante del uso de una tecnología (Mouakket, S. y Bettayeb, A. M., 2015; Chang, S. C. y Tung, F. C., 2008). Esto indica que el e-learning puede considerarse como una herramienta de la tecnología de la información. Pero, solamente será aceptado por los estudiantes cuando perciban que su uso va a mejorar su rendimiento en el aprendizaje, como fue comprobado en varios estudios (Salloum, S. A. S., 2018; Sharma, S., Hasteer, N., Mishra, S. P. y Van Belle, J. P., 2016). Consecuentemente, en este contexto, la utilidad percibida del e-learning puede ser definida como el grado en que los estudiantes consideran que su uso optimizará el rendimiento en el aprendizaje. Por consiguiente, tendría un impacto en su intención de aceptar y adoptar directa o indirectamente dicho comportamiento.

\section{Facilidad de uso percibida}

La facilidad de uso percibida se define como el grado en que una 
persona cree que el uso de una tecnología específica es fácil de entender y libre de esfuerzo (Cheng, Y.M., 2011). De igual forma tiene relación positiva con la Intención de comportamiento (Cheng, B., Wang, M., Moormann, J., Olaniran, B. A. y Chen, N. S., 2012). Con respecto al e-learning, la facilidad de uso percibida se podría definir como la medida en que un estudiante considera que el uso del sistema de aprendizaje electrónico será fácil de usar y no requerirá mucho esfuerzo.

\section{Actitud}

Actitud se refiere al grado en que una persona tiene un sentimiento positivo o negativo hacia los sistemas de aprendizaje electrónico (EscobarRodriguez, T. y Monge-Lozano, P., 2012). Entonces, se entiende que la actitud es fundamental y siempre involucra una evaluación de forma favorable o desfavorable hacia un objeto, un concepto, una acción, entre otros. En este caso, valora una evaluación a los sistemas de aprendizaje electrónico. Varios estudios han demostrado que la actitud tiene un impacto directo en la intención de comportamiento (Farahat,
T., 2012). Por este motivo, cuanto más favorable sea la actitud hacia el e-learning, más probable será que la persona tenga intención de involucrarse en este comportamiento específico.

\section{Intención de comportamiento}

La intención de comportamiento se refiere a la voluntad individual de completar un comportamiento particular (Ajzen, I., 1991; Ajzen, J. y Fishbein M., 1980). Este concepto se puede aplicar en cualquier área de estudio de manera general. A continuación, se presenta un concepto en relación al e-learning. Fathema, Shannon y Ross definen a la intención de comportamiento en el ámbito del e-learning como la intención de los estudiantes en pro de emplear sistemas de aprendizaje electrónico y que implica el uso persistente desde el presente hasta el futuro. Fathema, N., Shannon, D. y Ross, M., 2015). En ese sentido, la intención de comportamiento refleja la predisposición de hacer uso de algún tipo de sistema de aprendizaje tecnológico y así mismo, una constancia en su utilización en el futuro. 


\section{Teoría del comportamiento planeado} (TPB)

Propuesta por Ajzen, la TPB es una extensión de la Teoría de Acción Razonada (TRA) (Ajzen, I., 1985; Ajzen, J. y Fishbein M., 1980). Este modelo está basado en la integración de otras variadas teorías de la actitud (teorías del aprendizaje, teorías de valorexpectativas, teorías de consistencia y teoría de atribuciones). El modelo sugiere que las personas están más cerca de desarrollar intenciones cuando tienen una actitud positiva hacia sus pares; además, esperan que se realice este comportamiento de manera adecuada. Por otro lado, la TPB se enfoca en situaciones cuando los individuos carecen del control completo sobre su comportamiento (Ajzen, J. y Fishbein M., 1980). Los múltiples constructos incluidos en la TPB son las actitudes comportamentales, las normas subjetivas, la intención de uso y el uso actual. Más aún, la TPB también comprende un constructo de percepción denominado Control percibido del comportamiento. Tomando en cuenta lo anterior, el aspecto del control de la observación hace que la TPB sea un modelo más funcional que la Teoría de la acción razonada, para su aplicación en cualquier ámbito de estudio. Sin embargo, se observa que la TPB es usada ampliamente para analizar la aceptación de una variedad de nuevos productos de tecnologías de la información y también al nivel de predicción de uso de estos (Pattansheti, M., Kamble, S. S., Dhume, S. M. y Raut, R. D., 2016; Issa, I. y Hamm, U., 2017; Xie, Q., Song, W., Peng, X. y Shabbir, M., 2017; Ma, J., Hipel, K. W., Hanson, M. L., Cai, X. y Liu, Y., 2018). Como se mencionó anteriormente, la TPB es usada ampliamente; no obstante, existen áreas donde es más aplicada que en otras por su nivel de efectividad y predicción, como es el caso de las tecnologías de información. En la Figura 2 , se muestra el modelo original de la TPB. 


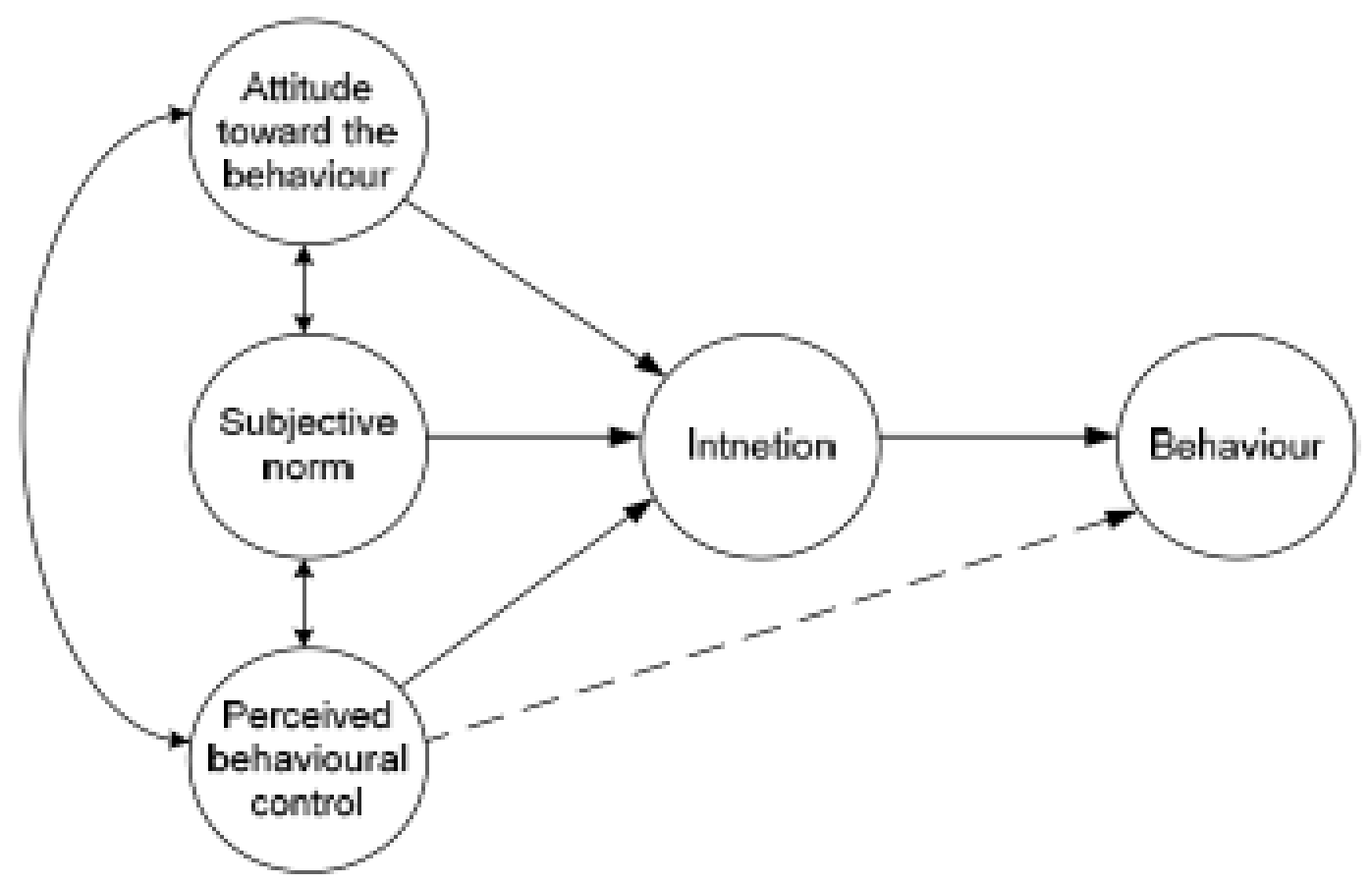

Figura 2. Modelo TPB. (Fuente: Ajzen, 1991)

\section{Actitud}

De acuerdo con Ajzen, este constructo se refiere al grado en que una persona tiene una evaluación favorable o desfavorable del comportamiento en cuestión. Además incluye un juicio sobre si el comportamiento que se considera es bueno o malo, y si la persona quiere realizarlo o no (Ajzen, 1991; Leonard, M., Graham, S. y Bonacum, D., 2004). Tomando en cuenta la afirmación anterior, con respecto al e-learning, la actitud se refiere al resultado de las creencias y evaluación de los estudiantes hacia el uso y aplicación de esta formación o enseñanza online. Si los estudiantes forman una actitud positiva hacia este, tendrán una intención más fuerte hacia su adopción y, por ello, es más probable que lo utilicen (Hong, S., Thong, J. Y. L. y Tam, K. Y. 2006; Hsu, M. H., Yen, C. H., Chiu, C. M. y Chang, C. M., 2006). Los antecedentes del constructo de actitud son denominadas creencias de conductuales o de actitud. Tomando en cuenta las investigaciones de Davis y Teo, quienes argumentan las relaciones causales entre la facilidad de uso, la utilidad percibida, la actitud y la intención de uso, se consideran como creencias de actitud a la facilidad de uso y la utilidad percibida. 


\section{Norma subjetiva}

Ajzen la define como "presión social percibida para realizar o no el un comportamiento particular" (Ajzen, I., y Driver, B.L., 1992: 208). Se cree que es un factor social en la naturaleza. La norma subjetiva capta los sentimientos de los individuos sobre la presión social que sienten sobre un determinado comportamiento. En consecuencia, si los consumidores tienen sentimientos positivos hacia un comportamiento dado, las intenciones en cuestión tienen más probabilidades de aumentar (Han, H. y Kim, Y., 2010; Taylor, S. y Todd, P., 1995). Debido a esto, muchas personas eligen utilizar determinados productos $\mathrm{o}$ servicios porque sus amigos o familiares ya lo han utilizado y se lo recomiendan. Varios estudios han documentado la norma subjetiva como un determinante importante para predecir el comportamiento y la intención del uso en distintas áreas, como educación, tecnología, entre otras (Baker, E. W., AlGahtani, S. S. y Hubona, G. S. 2007; Venkatesh, V. y Davis, F. D., 2000). Por este motivo, existe especial atención en la norma subjetiva, indistintamente del área de estudio ya que, al momento de predecir un comportamiento, sus resultados son específicos y elevados.

La norma subjetiva al igual que la actitud está determinada por creencias, en este caso creencias normativas y pueden descomponerse en múltiples grupos de referencia con diferentes puntos de vista (Ajzen, I., 1991; Taylor, S. y Todd, P., 1995). Como estos autores explican, las creencias normativas pueden contemplar varios grupos de referencia (familia, amigos, colegas, entre otros) desde distintos puntos de vista; estos pueden ser tomados en cuenta de acuerdo al tipo de área y tema de estudio. Estudios referentes al tema de investigación sugieren dos grupos de referencia relevantes en la educación superior: estudiantes y docentes (Liu, Y. y Chen, N. S., 2008; Taylor, S. y Todd, P. (1995). Por lo tanto, se consideran como antecedentes de la norma subjetiva la preparación del estudiante $\mathrm{y}$ del docente.

\section{Control conductual}

El control conductual diferencia la TPB respecto a la TRA y se define como la facilidad o dificultad percibidas por un 
individuo para realizar el importante (Liaw, S. S., Huang, H. M. y comportamiento en particular (Ajzen, I., 1991). En otras palabras, el control conductual mide la percepción de un individuo en relación a que es suficientemente hábil, disciplinado y capaz de llevar a cabo un determinado comportamiento. En este caso, aunque el e-learning es un instrumento útil para mejorar el aprendizaje, se necesitan conocimientos básicos de internet para su uso de manera eficiente. Así mismo Ajzen, Fishbein y Cappella afirman que el comportamiento conductual deriva del concepto de autoeficacia (Ajzen, I., 1991; Fishbein, M. y Ajzen, J., 1975; Fishbein, M. y Cappella, J., 2006), que hace referencia a las creencias de los individuos sobre su capacidad $\mathrm{y}$ motivación para realizar tareas específicas (Bandura, A., 1986). En estudios anteriores, se ha comprobado que niveles más altos de autoeficacia conducen a niveles más altos de intención conductual y al uso de la tecnología de la información (Compeau, D. R. y Higgins, C. A., 1995; Gist, M. E., Schwoerer, C. y Rosen, B., 1989). Como segundo antecedente, se tomó la autonomía de aprendizaje, antecedente que ha demostrado ser también un factor

\section{Adaptación del aprendizaje virtual}

Es un indicador de la disposición del individuo para realizar un comportamiento dado y antecede al constructo final (Ajzen, I., 2002). Cuanto más favorable sea la actitud hacia el comportamiento, más favorable sea la norma subjetiva; cuanto mayor sea el control conductual, más fuerte será la intención del individuo de realizar el comportamiento, en este caso el uso de $e$ learning.

\section{E-learning}

"E-learning puede entenderse como un proceso educativo que utiliza las tecnologías de la información y las comunicaciones para crear capacitación, distribuir el contenido del aprendizaje, la comunicación entre estudiantes y profesores y para la gestión de los estudios" (Wagner, E. D., 2005: 41). En otras palabras, para mejorar el 
aprendizaje online, este proceso educativo aprovecha la tecnología disponible y gestiona el contenido a aprender, así como la relación entre los involucrados. El e-learning desafía las formas tradicional aprendizaje, proporcionando nuevas soluciones a los problemas, es activo y acorde al ritmo de cada individuo (Haverila, M. y Barkhi, R., 2009; Obringer Lee, Ann, 2002). Es decir, el $e$ learning es muy versátil; nos muestra distintas respuestas a los problemas adaptándose a cada persona. Contiene diferentes tipos de herramientas educativas y puede referirse a diferentes entornos de aprendizaje, en distintos niveles educativos $y$ formatos de presentación; ofrece muchas oportunidades para todo los involucrados electrónicos (Cheng, Y.M., 2012; Holmes, B. y Gardner, J., 2006). Esto nos da la posibilidad de aprender a la hora y en el lugar que uno quiera, sin ningún problema o limitación, desde cualquier parte del mundo.

\section{Modelo teórico e hipótesis}

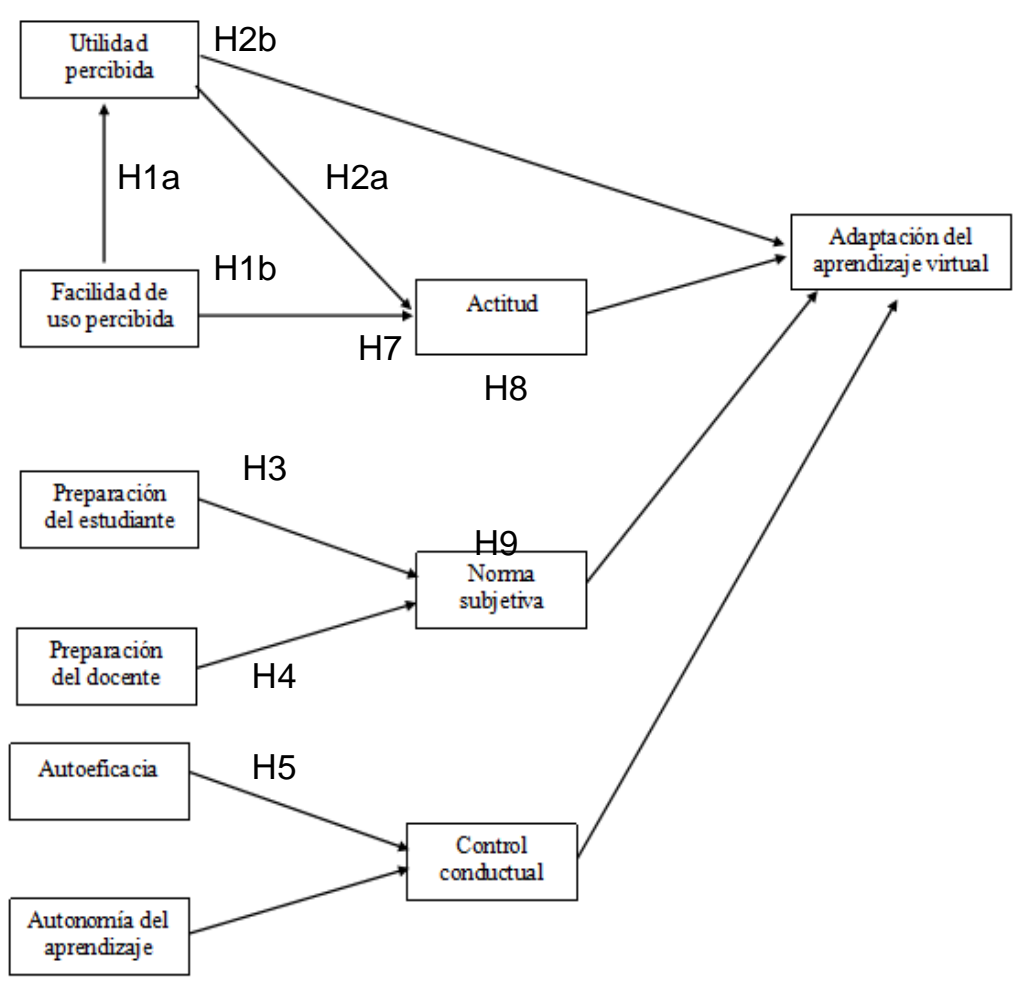

Figura 3. Modelo unificado. (Fuente: Elaboración propia, 2020) 
H1a: La facilidad de uso percibida tiene influencia positiva en la utilidad percibida

H1b: La facilidad de uso percibida tiene influencia positiva en la actitud.

H2a: La utilidad percibida tiene influencia positiva en la actitud.

H2b: La utilidad percibida tiene influencia positiva en la intención de comportamiento.

H3: La preparación del estudiante tiene influencia positiva en la norma subjetiva.

H4: La preparación del docente tiene influencia positiva en norma subjetiva.

H5: La autoeficacia tiene influencia positiva en el control conductual.

H6: La autonomía del aprendizaje tiene influencia positiva en el control conductual.

H7: La actitud hacia tiene influencia positiva en la adaptación del aprendizaje virtual.

H8: La norma subjetiva tiene influencia positiva en la adaptación del aprendizaje virtual.

\section{RESULTADOS Y DISCUSIÓN}

El análisis se realizó con un modelo consistente de ecuaciones estructurales de mínimos cuadrados parciales (PLSc SEM). Dijkstra y Henseler establecieron que PLSc-SEM es menos sensible a los errores de Tipo I y Tipo II que PLS-SEM (Dijkstra, T. K. y Henseler, J., 2015); puede utilizarse con modelos en los que todos los constructos sean reflectivos como es el caso en la presente investigación. Los resultados generados por la ejecución del algoritmo PLSc se evalúan en dos etapas: según Barclay, Higgins y Thompson): modelo de medida y modelo estructural (Barclay. D. W., Higgins. C. A. y Thompson. R., 1995). Por medio de estas etapas se puede asegurar y la validar la calidad de los datos y resultados proporcionados los el software utilizado. Para los modelos reflectivos, como los de la presente investigación se toman en cuenta los siguientes criterios: 
- Fiabilidad de los indicadores,

- Consistencia interna,

- Validez convergente y

- Validez discriminante.

La fiabilidad de los elementos se mide a través de las cargas factoriales, se recomienda que las cargas de factor excedan de 0.7. Generalmente los indicadores con cargas entre 0.4 y 0.70 deben considerarse para su eliminación solo cuando ésta lleve a un aumento de la fiabilidad compuesta o de la varianza media extraída por encima del valor umbral sugerido. Nunnally y Bernstein sugieren validar estos indicadores con un valor de al menos 0.7 , considerado como un nivel "modesto" principalmente para investigaciones exploratorias (Nunnally, J. y Bernstein, I., 1994). Para verificar la consistencia de los indicadores, se analizaron Alfa de Cronbach, Coeficiente Spearman (rho_A) y la fiabilidad compuesta. Analizando la validez convergente mediante la varianza media extraída (AVE), cuyo valor recomendable es mayor a 0.5. Si este valor es menor en algún indicador, pero la fiabilidad compuesta es a mayor 0.6 ; la validez convergente del constructo puede ser aceptable (Fornell, C. y Larcker, D. (1981). Por último, la validez discriminante fue analizada mediante el Criterio de Fornell-Lacrker, el cual explica que la cantidad de varianza que un constructo captura de sus indicadores debe ser mayor a la varianza que el constructo comparte con otros constructos. Además, el criterio HTMT que representa el promedio de las correlaciones entre los indicadores que miden en mismos constructos con relación a los que miden diferentes constructos, este valor debe estar por debajo de 0.90. La evaluación del modelo se realizó tomando en cuenta estas etapas y criterios de evaluación. 


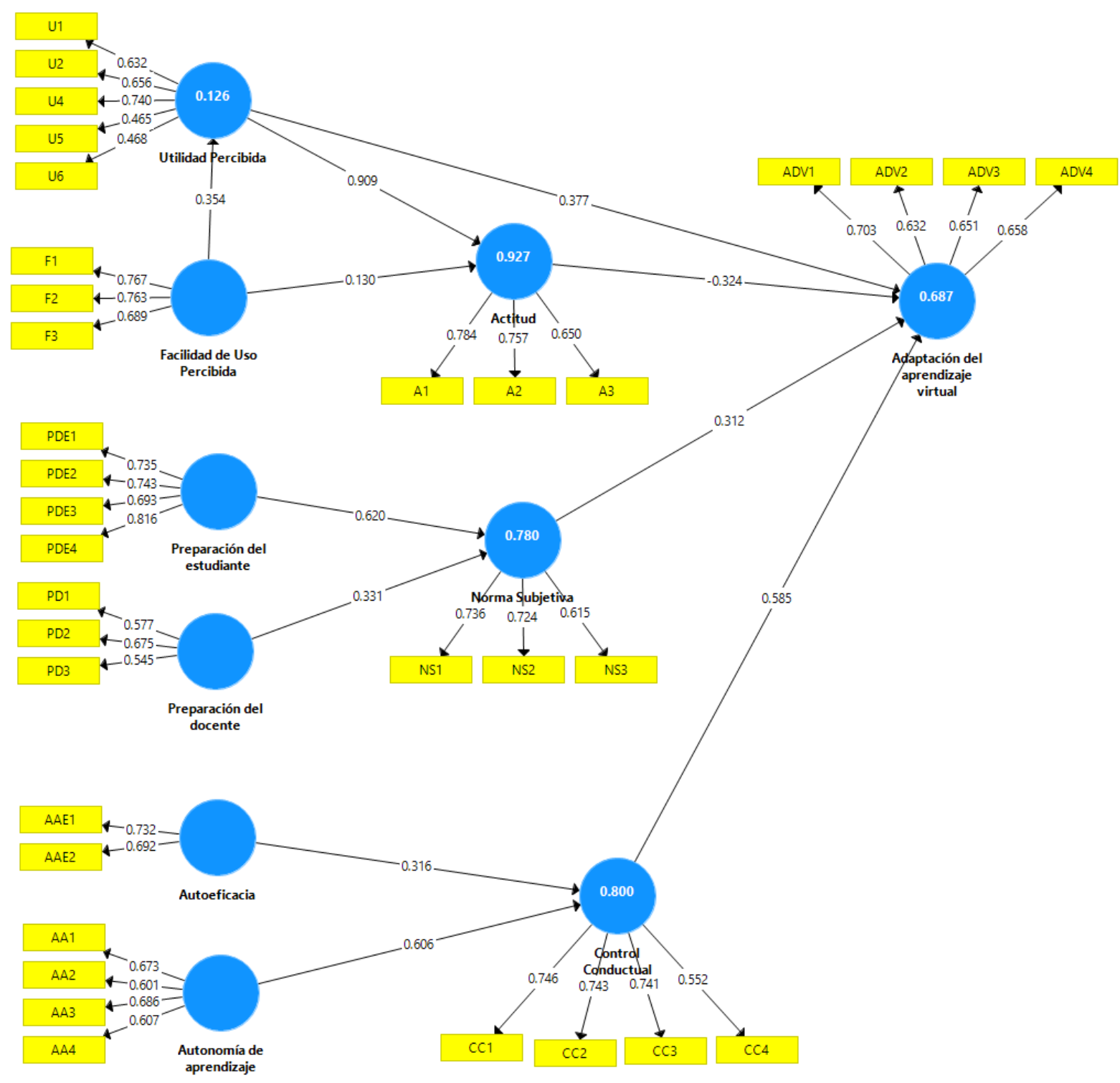

Figura 4. Modelo Integrado. (Fuente: Elaboración propia, 2020 -con Smart PLS-)

\section{Modelo de medida}

Para los modelos reflectivos como los de la presente investigación se tomaron en cuenta los siguientes criterios:

- Fiabilidad de los indicadores,

- Consistencia interna,

- Validez convergente y

- Validez discriminante. 


\section{Validación de la fiabilidad de los indicadores}

Como se observa en las Tabla 2, los indicadores que fueron eliminados debido a que las cargas que presentaban eran menores a 0.4 .

Tabla 2. Indicadores eliminados Modelo Integrado

\begin{tabular}{ll}
\hline Constructo & Indicador \\
\hline Autoeficacia & AAE3 \\
& AAE4 \\
Utilidad percibida & U3 \\
Preparación del docente & PD4 \\
\hline
\end{tabular}

Fuente: Elaboración propia, 2020 (con Smart PLS)

Consistencia interna $\mathbf{y}$ validez pertenecientes a los distintos constructos convergente oscilan entre 0.465 y 0.816 . En su mayoría, los constructos cumplen con los

Una vez eliminados los indicadores, valores umbrales den todos los criterios. se evaluó la consistencia interna y validez Si bien la varianza extraída media en convergente de ambos modelos algunos constructos es menor al valor propuestos. La Tabla 3 hace referencia a umbral 0.5, la fiabilidad compuesta en los criterios de evaluación del modelo todos los constructos es mayor a 0.6. integrado, en el cual se observa que las Entonces, los valores se consideran cargas factoriales de los indicadores aceptables. 
Tabla 3. Fiabilidad del constructo Modelo Integrado

\begin{tabular}{|c|c|c|c|c|c|}
\hline Constructo & $\begin{array}{l}\text { Cargas } \\
\text { factoriales }\end{array}$ & $\begin{array}{l}\text { Alfa de } \\
\text { Cronbach }\end{array}$ & rho_A & $\begin{array}{l}\text { Fiabilidad } \\
\text { compuesta }\end{array}$ & $\begin{array}{c}\text { Varianza } \\
\text { extraída } \\
\text { media }\end{array}$ \\
\hline Actitud (A) & $0.650-0.784$ & 0.774 & 0.781 & 0.775 & 0.537 \\
\hline $\begin{array}{c}\text { Adaptación del } \\
\text { aprendizaje virtual } \\
\text { (ADV) }\end{array}$ & $0.632-0.703$ & 0.757 & 0.758 & 0.757 & 0.438 \\
\hline Autoeficacia (AAE) & $0.692-0.732$ & 0.672 & 0.674 & 0.673 & 0.507 \\
\hline $\begin{array}{c}\text { Autonomía de } \\
\text { aprendizaje(AA) }\end{array}$ & $0.601-0.686$ & 0.737 & 0.740 & 0.737 & 0.413 \\
\hline Control conductual (CC) & $0.552-0.746$ & 0.775 & 0.802 & 0.792 & 0.491 \\
\hline $\begin{array}{l}\text { Facilidad de uso } \\
\text { percibida }(\mathrm{F})\end{array}$ & $0.689-0.767$ & 0.785 & 0.786 & 0.784 & 0.548 \\
\hline Norma subjetiva (NS) & $0.615-0.736$ & 0.726 & 0.740 & 0.734 & 0.481 \\
\hline $\begin{array}{l}\text { Preparación del docente } \\
\text { (PD) }\end{array}$ & $0.545-0.675$ & 0.613 & 0.634 & 0.628 & 0.362 \\
\hline $\begin{array}{l}\text { Preparación del } \\
\text { estudiante (PDE) }\end{array}$ & $0.693-0.816$ & 0.833 & 0.838 & 0.835 & 0.560 \\
\hline Utilidad percibida (U) & $0.465-0.740$ & 0.712 & 0.754 & 0.733 & 0.363 \\
\hline
\end{tabular}

*Nota: rho_A. Coeficiente de correlación de Spearman. (Fuente: Elaboración propia, 2020 -con Smart PLS-)

\section{Validez discriminante}

La validez discriminante se evaluó en base a dos criterios:

- Criterio de Fornell-Larcker y

- Matriz HTMT.

Tabla 1. Criterio de Fornell-Larcker Modelo Integrado

\begin{tabular}{lcccccccccc}
\hline & A & ADV & AAE & AA & CC & F & NS & PD & PDE & U \\
\hline A & 0.732 & & & & & & & & & \\
ADV & 0.027 & 0.662 & & & & & & & & \\
AAE & -0.074 & 0.702 & 0.712 & & & & & & & \\
AA & 0.008 & 0.834 & 0.868 & 0.643 & & & & & & \\
CC & -0.067 & 0.770 & 0.842 & 0.881 & 0.700 & & & & & \\
F & 0.452 & -0.013 & -0.101 & -0.066 & -0.059 & 0.740 & & & & \\
NS & 0.096 & 0.733 & 0.756 & 0.801 & 0.675 & -0.009 & 0.693 & & & \\
PD & 0.044 & 0.867 & 0.775 & 0.832 & 0.826 & -0.093 & 0.763 & 0.601 & & \\
PDE & 0.073 & 0.588 & 0.706 & 0.782 & 0.646 & -0.057 & 0.851 & 0.698 & 0.748 & \\
U & 0.955 & 0.042 & -0.116 & -0.010 & -0.124 & 0.354 & 0.152 & 0.030 & 0.086 & 0.60 \\
\hline
\end{tabular}

Fuente: Elaboración propia, 2020 (con Smart PLS) 
En la Tabla 4 se observa que el discriminatoria suficiente, a pesar de que Criterio de Fornell-Larcker no se cumple no se cumpla el criterio de Fornellen ningún constructo (Henseler, J., Larcker, siempre que la correlación Ringle, C. M. y Sarstedt, M., 2015). No ～(criterio HTMT) entre los factores no sea obstante, puede asumirse una validez superior a 0.9.

Tabla 5. Criterio HTMT Modelo Integrado

\begin{tabular}{lcccccccccc}
\hline & A & ADV & AAE & AA & CC & F & NS & PD & PDE & U \\
\hline A & & & & & & & & & \\
ADV & 0.113 & & & & & & & & & \\
AAE & 0.088 & 0.700 & & & & & & & & \\
AA & 0.093 & 0.833 & 0.868 & & & & & & & \\
CC & 0.090 & 0.796 & 0.853 & 0.892 & & & & & & \\
F & 0.459 & 0.082 & 0.103 & 0.075 & 0.107 & & & & \\
NS & 0.113 & 0.745 & 0.762 & 0.809 & 0.708 & 0.030 & & & & \\
PD & 0.109 & 0.887 & 0.786 & 0.846 & 0.868 & 0.105 & 0.785 & & & \\
PDE & 0.078 & 0.590 & 0.711 & 0.787 & 0.664 & 0.082 & 0.857 & 0.714 & & \\
U & 0.976 & 0.074 & 0.119 & 0.099 & 0.145 & 0.375 & 0.162 & 0.058 & 0.108 & \\
\hline
\end{tabular}

Fuente: Elaboración propia, 2020 (con Smart PLS)

Como se puede observar en la Tabla 5 , las relaciones entre la mayoría de los constructos presentan valores menores a 0.9, a excepción de los constructos Utilidad percibida y Actitud. Consecuentemente, se procedió a realizar un HTMT inferencial el cual debe presentar valores menores a 1.00
(Henseler, J., Ringle, C. M. y Sarstedt, M., 2015). Para conformar o no la existencia de validez discriminante en el modelo propuesto, el valor HTMT inferencial de la relación de los constructos mencionados dio un valor de 1.037. Ello confirma que no existe validez discriminante entre los mismos. 


\section{Modelo estructural}

Una vez evaluado el modelo de medida se procede a evaluar el modelo estructural; este se lleva a cabo a través de:

- Coeficiente de determinación $\left(\mathrm{R}^{2}\right)$,

- Coeficientes pathy

- Ajuste del modelo.

\section{Coeficiente de determinación}

Al momento de analizar el coeficiente de determinación $\left(\mathrm{R}^{2}\right)$, se llega a conocer la cantidad de varianza de un constructo endógeno que es explicada por las variables predictoras del mismo; los valores se encuentran entre 0 y 1 ; cuanto más cerca de 1 , mayor será la capacidad predictiva del modelo para dicho constructo (Roldán, J. L. y Cepeda, G., 2016). Cabe también recordar en esta oportunidad que Chin Chin, W. W. (1998) considera 0.67 como sustancial, 0.33 moderado y 0.19 débil; mientras que Hair Jr, J. F., Hult, G. T. M., Ringle, C. y Sarstedt, M. (2014) recomiendan 0.75 sustancial, 0.50 moderado y 0.25 débil.

Tabla 6. Coeficiente de determinación

\begin{tabular}{ll}
\hline Variable & $\mathbf{R}^{2}$ \\
\hline Utilidad percibida (U) & 0.126 \\
Actitud (A) & 0.927 \\
Control conductual (CC) & 0.800 \\
Norma subjetiva (NS) & 0.780 \\
Adaptación del aprendizaje virtual (ADV) & 0.687 \\
\hline
\end{tabular}

Fuente: Elaboración propia, 2020 (con Smart PLS)

En la Tabla 6, se puede observar que en su mayoría los constructos presentan una relación sustancial, a excepción del constructo Utilidad percibida, cuya relación no pasa el valor umbral para ser considerado mínimamente como débil. El constructo con mayor explicación por parte de sus variables es el de Actitud seguido por Control conductual. A continuación, se realiza la descomposición del coeficiente de determinación, para conocer el porcentaje de explicación de las variables predictoras a cada constructo. 
Tabla 7. Descomposición del coeficiente de determinación de Utilidad percibida

\begin{tabular}{cccc}
\hline Constructos & $\begin{array}{c}\text { Coeficiente de } \\
\text { correlación }\end{array}$ & $\begin{array}{c}\text { Coeficiente } \\
\text { path }\end{array}$ & $\begin{array}{c}\text { Porcentaje } \\
\text { explicado }\end{array}$ \\
\hline Facilidad de uso percibida & 0.354 & 0.354 & $12.53 \%$ \\
\hline
\end{tabular}

Fuente: Elaboración propia, 2020 (con Smart PLS)

Como se puede observar en la Tabla 7, el constructo Utilidad percibida es explicado en un $12.53 \%$ por la Facilidad de uso percibida y el porcentaje restante explicado por variables que no fueron estudiadas.

Tabla 8. Descomposición del coeficiente de determinación de Actitud

\begin{tabular}{lccc}
\hline \multicolumn{1}{c}{ Constructos } & $\begin{array}{c}\text { Coeficiente de } \\
\text { correlación }\end{array}$ & $\begin{array}{c}\text { Coeficiente } \\
\text { path }\end{array}$ & $\begin{array}{c}\text { Porcentaje } \\
\text { explicado }\end{array}$ \\
\hline Utilidad percibida & 0.955 & 0.909 & $86.80 \%$ \\
Facilidad de uso percibida & 0.452 & 0.130 & $6.00 \%$ \\
\hline
\end{tabular}

Fuente: Elaboración propia, 2020 (con Smart PLS)

La Tabla 8 referente al constructo Actitud es explicada en su mayoría por la Utilidad percibida, en un $86.80 \%$ y por un porcentaje mínimo de $6.00 \%$ por la Facilidad de uso percibida.

Tabla 9. Descomposición del coeficiente de determinación de Norma subjetiva

\begin{tabular}{lccc}
\hline \multicolumn{1}{c}{ Constructos } & $\begin{array}{c}\text { Coeficiente de } \\
\text { correlación }\end{array}$ & $\begin{array}{c}\text { Coeficiente } \\
\text { path }\end{array}$ & $\begin{array}{c}\text { Porcentaje } \\
\text { explicado }\end{array}$ \\
\hline Preparación del estudiante & 0.851 & 0.620 & $52.80 \%$ \\
Preparación del docente & 0.763 & 0.330 & $25.20 \%$ \\
\hline
\end{tabular}

Fuente: Elaboración propia, 2020 (con Smart PLS)

Como se puede observar en la Tabla 9, el constructo Norma subjetiva es explicado en $52.80 \%$ por la Preparación del estudiante y, en $25.20 \%$, por la Preparación del docente. 
Tabla 10. Descomposición del coeficiente de determinación de Control conductual

\begin{tabular}{lccc}
\hline Constructos & $\begin{array}{c}\text { Coeficiente de } \\
\text { correlación }\end{array}$ & $\begin{array}{c}\text { Coeficiente } \\
\text { path }\end{array}$ & $\begin{array}{c}\text { Porcentaje } \\
\text { explicado }\end{array}$ \\
\hline Autonomía de aprendizaje & 0.881 & 0.606 & $53.40 \%$ \\
Autoeficacia & 0.842 & 0.316 & $26.60 \%$ \\
\hline
\end{tabular}

Fuente: Elaboración propia, 2020 (con Smart PLS)

En la Tabla 10, se puede observar que el constructo Control conductual es explicado en $53.40 \%$ por la Autonomía de aprendizaje y, en $26.60 \%$, por la Autoeficacia.

Tabla 11. Descomposición del coeficiente de determinación de Adaptación del aprendizaje virtual

\begin{tabular}{lccc}
\hline \multicolumn{1}{c}{ Constructos } & $\begin{array}{c}\text { Coeficiente de } \\
\text { correlación }\end{array}$ & $\begin{array}{c}\text { Coeficiente } \\
\text { path }\end{array}$ & $\begin{array}{c}\text { Porcentaje } \\
\text { explicado }\end{array}$ \\
\hline Utilidad percibida & 0.042 & 0.377 & $1.58 \%$ \\
Actitud & 0.027 & -0.324 & $-0.87 \%$ \\
Norma subjetiva & 0.733 & 0.312 & $22.87 \%$ \\
Control conductual & 0.770 & 0.585 & $45.05 \%$ \\
\hline
\end{tabular}

Fuente: Elaboración propia, 2020 (con Smart PLS)

Por último, en la Tabla 11, se puede observar que la Adaptación del aprendizaje virtual es explicada en $45.05 \%$ por el Control conductual; en un $22.87 \%$, por la Norma subjetiva y, en un porcentaje mínimo de $1.58 \%$, por la Utilidad percibida. 


\section{Coeficientes path}

Tabla 12. Resultado del modelo estructural Integrado

\begin{tabular}{lcccc}
\hline Constructos & Coeficiente path & Estadísticos t & P value & $\begin{array}{c}\text { Comprobación de } \\
\text { hipótesis }\end{array}$ \\
\hline H1. F $\rightarrow$ A & 0.130 & 1.953 & 0.051 & No Soportada \\
H2. F $\rightarrow$ U & 0.354 & 3.812 & 0.000 & Soportada \\
H3 .U $\rightarrow$ A & 0.909 & 21.721 & 0.000 & Soportada \\
H4. A $\rightarrow$ ADV & -0.324 & 0.001 & 0.999 & No Soportada \\
H5. AA $\rightarrow$ CC & 0.606 & 0.184 & 0.854 & No Soportada \\
H6. PD $\rightarrow$ NS & 0.331 & 2.508 & 0.012 & Soportada \\
H7. NS $\rightarrow$ ADV & 0.312 & 0.008 & 0.993 & No Soportada \\
H8. CC $\rightarrow$ ADV & 0.585 & 0.010 & 0.992 & No Soportada \\
H9. PDE $\rightarrow$ NS & 0.620 & 5.102 & 0.000 & Soportada \\
H10. AAE $\rightarrow$ CC & 0.316 & 0.096 & 0.923 & No soportada \\
H11. U $\rightarrow$ ADV & 0.377 & 0.001 & 0.999 & No Soportada \\
\hline
\end{tabular}

Fuente: Elaboración propia, 2020 (con Smart PLS)

\section{Implicaciones gerenciales}

El análisis de ImportancePerformance Map Analysis (IPMA) permite identificar cuáles variables (constructos o indicadores) resultan más importantes, es decir aquellos con mayor efecto total en el constructo de preferencia y también aquellos con menor rendimiento en el mismo (Hair, J. F., Hult, G. T., Ringle, C. M., Sarstedt, M., Castillo Apraiz, J., Cepeda Carrión, G. y Roldan, J. L., 2019; Hock, C., Ringle, C. M. y Sarstedt, M., 2010; y
Ringle, C. M. y Sarstedt, M., 2016). Los factores de prioridad y con características más importantes están en el cuadrante inferior derecho (I); sin embargo, presentan bajo desempeño; los resultados en el cuadrante inferior izquierdo (II) explican las características menos importantes y con bajo rendimiento; el cuadrante superior izquierdo refleja los atributos menos importantes y con rendimiento alto, por último, el cuadrante superior derecho muestra atributos importantes y con mayor rendimiento (Melo, A., Ayaviri- 
Panozo, A., y Rocha, M., 2018). En este caso, se realiza un análisis IPMA de los construntos antecesores directos e indirectos para el constructo objetivo Adaptación del apendizaje virtual. Se toman en cuenta los antecedentes directos e indirectos a este. Los resultados de la matriz IPMA de indicadores se muestra a continuacion en la Figura 5. Tomando en cuenta el valor promedio de los efectos y rendimientos totales hacia el constructo Adaptación del aprendizaje virtual, se pudo realizar los puntos de corte en el eje X; el valor promedio del rendimiento es de 68.6 y en el eje $\mathrm{Y}$ el valor promedio de los efectos totales es de 0.147 .

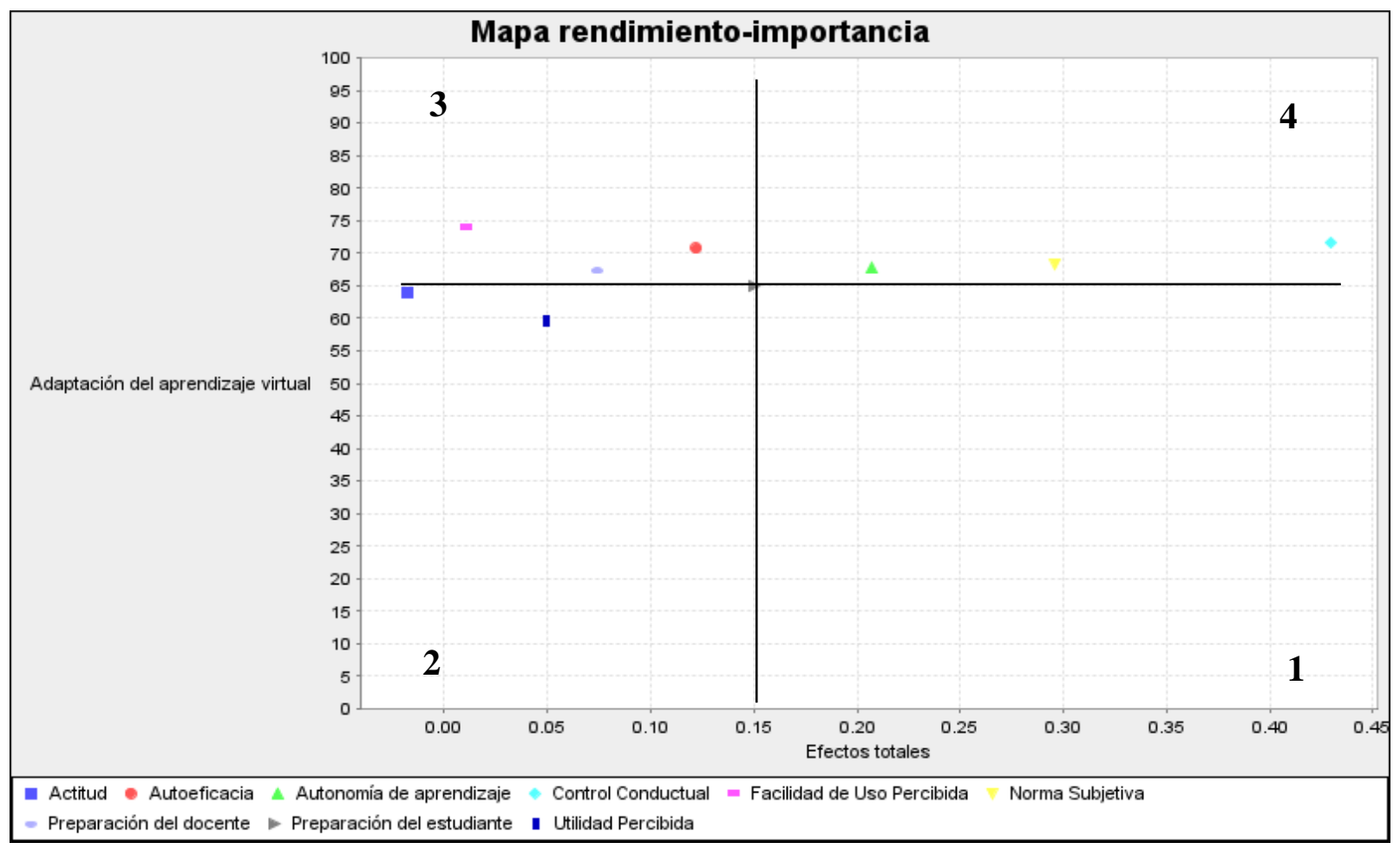

Figura 5. Matriz IPMA. (Fuente: Elaboración propia, 2020 -con Smart PLS-)

Como se puede observar en la importantes, pero un bajo desempeño; Figura 5, los constructos se encuentran en el segundo cuadrante, se observan los distribuidos en los cuatro cuadrantes. En constructos Utilidad percibida y Actitud, el primer cuadrante, se halla el que explican características menos constructo Preparación del estudiante, importantes y tienen un bajo un constructo con características rendimiento. En el tercer cuadrante, se 
advierten los indicadores de Autoeficacia, Preparación docente y Facilidad de uso percibida; son menos importantes, empero poseen un alto rendimiento. Por último, el cuarto cuadrante refleja indicadores importantes (Control conductual, Norma subjetiva y Autonomía de aprendizaje), con un rendimiento y desempeño elevados. Por lo tanto, se deberían plantear estrategias orientadas a estos tres últimos para así aumentar la Adaptación de aprendizaje virtual.

\section{CONCLUSIONES}

Gracias a la revisión de la literatura respecto al tema, se pudo identificar los factores prevalentes que impactan en el aprendizaje virtual. En este caso son los propuestos por los autores Ajzen (1991) y Davis (1985) cuyas teorías se aplican ampliamente en este ámbito. Mediante la revisión meta-analítica, se pudo recabar información relevante para el presente estudio. Ello permitió la combinación varios modelos sobre los factores de impacto en el aprendizaje virtual. A través del uso del software SmartPLS, usando la técnica de ecuaciones estructurales, se pudo validar el modelo adoptado tomando en cuenta varios criterios de evaluación, así como la comprobación de las hipótesis planteadas, de las cuales siete fueron rechazadas, como pudo observarse anteriormente en la Tabla 12. Mediante el análisis de la matriz de importanciarendimiento, se pudo medir el valor de la influencia de las variables que generan un mayor impacto en el aprendizaje virtual.

Se puede concluir que la actitud de los participantes frente al aprendizaje virtual es que ellos consideran que el aprendizaje virtual no es bueno, positivo ni práctico. Como se observó en la Tabla 12 con la hipótesis número cuatro. Por su parte, el control conductual, hace referencia a que el estudiante cuenta con el suficiente conocimiento, control para la toma de decisiones, confianza en sí mismo y la interacción entre docentes y pares; se guarda una relación positiva respecto a la adopción del aprendizaje virtual.

Por último, la utilidad percibida del aprendizaje virtual, representada en el mejoramiento del desempeño académico, se refleja en un aumento de la productividad académica, facilidad en el estudio del contenido de los cursos, acceso en tiempo real y la mejora del contacto con los docentes, esta relación también es positiva respecto a la adopción del aprendizaje virtual. 


\section{REFERENCIAS}

Ajzen, I. (1985). From intentions to actions: a theory of planned behaviour. Action Control. Recuperado de https://doi.org/ 10.1007/978-3-642-69746-3_2)

Ajzen, I. (1991). The Theory of Planned Behavior. Organization Behavior and Human Decision Process. Recuperado de https://doi.org/10.1016/0749-5978 (91)90020-T

Ajzen, I. (2002). Perceived behavioral control, self-efficacy, locus of control, and the Theory of Planned Behavior. Journal of Applied Social Psychology. Recuperado de https://doi.org/10.1111/j.1559-

1816.2002.tb00236.x

Ajzen, I., y Driver, B.L. (1992). Application of the theory of planned behavior to leisure choice. Journal of Leisure Research. Recuperado de https://doi.org/10.1080/00222216. 1992.11969889

Ajzen, J. y Fishbein M. (1980): Understanding attitudes and predicting social behavior. New Jersey, United States. Prentice-Hall

Baker, E. W., Al-Gahtani, S. S. y Hubona, G. S. (2007). The effects of gender and age on new technology implementation in a developing country. Information Technology \& People. Recuperado de https://doi. org/10.1108/09593840710839798

Bandura, A. (1986). The explanatory and predictive scope of self-efficacy theory. Journal of social and clinical psychology. Recuperado de http://dx.doi.org/10.1521/jscp.198 6.4.3.359

Barclay. D. W., Higgins. C. A. y Thompson. R. (1995). The partial least squares approach to causal modeling: personal computer adoption and use as an illustration. Technology studies: special issue on research methodology. Recuperado de http://people.stern.nyu.edu/ smezias/Using_Computer_Simulati ons.pdf

Bobsin, D., Visentini, M. S. y Rech, I. (2009). Em busca do estado da arte do UTAUT: ampliando as considerações sobre o uso da tecnologia. INMR-Innovation \& Management Review. Recuperado de https://doi.org/10.5773/ rai.v6i2.275

Carr Jr, V. H. (1999). Technology adoption and diffusion. The Learning Center for Interactive Technology. Recuperado de http://tlc.nlm.nih.gov/resources/pu blications/sourcebook/adoptiondiff usion.html

Chang, S. C. y Tung, F. C. (2008). An empirical investigation of students' behavioural intentions to use the online learning course websites. British Journal of Educational Technology. Recuperado de https://doi.org/10.1111/j.1467-

8535.2007.00742.x

Cheon, J., Lee, S., Crooks, S. M. y Song, J. (2012). An investigation of mobile learning readiness in higher education based on the theory of planned behavior. Computers \& Education. Recuperado de https://doi.org/10.1016/j.compedu .2012 .04 .015

Cheng, Y.M. (2011). Antecedents and consequences of e-learning acceptance. Information Systems Journal. Recuperado de https://doi.org/10.1111/j.1365-

2575.2010.00356.x 
Cheng, Y.M. (2012). Effects of quality antecedents on e-learning acceptance. Internet Research. Recuperado de https://doi.org/10. $1108 / 10662241211235699$

Cheng, B., Wang, M., Moormann, J., Olaniran, B. A. y Chen, N. S. (2012). The effects of organizational learning environment factors on elearning acceptance. Computers \& Education. Recuperado de https://doi.org/10.1016/j.compedu .2011.10.014

Cheung, R. y Vogel, D. (2013). Predicting user acceptance of collaborative technologies: An extension of the technology acceptance model for e-learning. Computers \& Education. Recuperado de https://doi.org/10. 1016/j.compedu.2012.12.003

Chin, W. W. (1998). The partial least squares approach to structural equation modeling. Modern methods for business research. Recuperado de https://www. researchgate.net/publication/31176 6005_The_Partial_Least_Squares_A pproach_to_Structural_Equation_M odeling

Chinyamurindi, W. y Shava, H. (2015). An investigation into e-learning acceptance and gender amongst final year students. South African Journal of Information Management. Recuperado de https://doi.org/10.4102/sajim.v17i 1.635

Cohen, J. (1988). Statistical power analysis for the behavioral sciences ( $2^{\text {da }}$ ed.). New Jersey: Erlbaum

Compeau, D. R. y Higgins, C. A. (1995). Computer self-efficacy: Development of a measure and initial test. Recuperado de https://doi.org/10.2307/249688

Davis, F. D. (1985). A technology acceptance model for empirically testing new end-user information systems: Theory and results (Doctoral dissertation,

Massachusetts Institute of Technology). Recuperado de https://dspace.mit.edu/bitstream/h andle/1721.1/15192/14927137MIT.pdf

Davis, F. D. (1989). Perceived usefulness, perceived ease of use, and user acceptance of information technology. Recuperado de https://doi.org/10.2307/249008

Davis, F. D., Bagozzi, R. P. y Warshaw, P.R. (1989). User acceptance of computer technology: A comparison of two theoretical models. Recuperado de https://doi. org $/ 10.1287 / \mathrm{mnsc} .35 .8 .982$

Davis, F. D., Bagozzi, R. P., y Warshaw, P. R. (1992). Extrinsic and intrinsic motivation to use computers in the workplace 1 . Recuperado de https://doi.org/10. 1111/j.1559-1816.1992.tb00945.x

Davis, F. D. y Venkatesh, V. (1996). A critical assessment of potential measurement biases in the technology acceptance model: three experiments. International Journal of Human-Computer Studies. Recuperado de https://doi.org/10. 1006/ijhc. 1996.0040

Dijkstra, T. K. y Henseler, J. (2015). Consistent partial least squares path modeling. Recuperado de https://doi.org/10.25300/MISQ/2 $015 / 39.2 .02$

Escobar-Rodríguez, T., y MongeLozano, P. (2012). The acceptance of Moodle technology by business administration students. Computers \& Education, 58(4), 1085-1093. Recuperado de https://doi.org/10. 1016/j.compedu.2011.11.012 
Farahat, T. (2012). Applying the technology acceptance model to online learning in the Egyptian universities. Procedia-Social and Behavioral Sciences. Recuperado de https://doi.org/10.1016/j.sbspro.20 12.11.012

Fathema, N., Shannon, D. y Ross, M. (2015). Expanding The Technology Acceptance Model (TAM) to Examine Faculty Use of Learning Management Systems (LMSs) In Higher Education Institutions. Journal of Online Learning \& Teaching. Recuperado de https://jolt.merlot.org/Vol11no2/F athema_0615.pdf

Fishbein, M. y Ajzen, J. (1975). Belief, attitude, intention, and behavior: an introduction to theory and research. United States, Addison-Wesley

Fishbein, M. y Capella, J. (2006). The role of theory in developing effective health communications. Journal of Communication. Recuperado de https://doi.org/ 10.1111/j.14602466.2006.00280.x

Fornell, C. y Larcker, D. (1981). Evaluating structural equation models with unobservable variables and measurement error. Journal of Marketing Research. Recuperado de https://doi.org/10.2307/3151312

Garavan, T., Carbery, R., O'Malley, G., y O'Donnell, D. (2010). Understanding participation in elearning in organizations: a largescale empirical study of employees. Recuperado de https://doi.org/10. 1111/j.1468-2419.2010.00349.x

Gefen, D., Karahanna, E. y Straub, D. (2003) Trust and TAM in online shopping: An integrated model. Recuperado de https://doi/10. 5555/2017181.2017185
Gist, M. E., Schwoerer, C. y Rosen, B. (1989). Effects of alternative training methods on self-efficacy and performance in computer software training. Journal of Applied Psychology. Recuperado de https://psycnet.apa.org/doi/10.103 7/0021-9010.74.6.884

Gist, M. E., Schwoerer, C. y Rosen, B. (1989). Effects of alternative training methods on self-efficacy and performance in computer software training. Journal of Applied Psychology. Recuperado de https://psycnet.apa.org/doi/10.103 7/0021-9010.74.6.884

$\mathrm{Gu}$, Ja-Chul y Lee, Sang-Chul y Suh, Yung, (2009). Determinants of behavioral intention to mobile banking. Expert Systems with Applications. Recuperado de https://doi.org/10.1016/j.eswa.200 9.03.024

Han, H. y Kim, Y. (2010). An investigation of green hotel customers' decision formation: Developing an extended model of the theory of planned behavior. Recuperado de https://doi.org/10.1016/j.ijhm.201 0.01 .001

Hair Jr, J. F., Hult, G. T. M., Ringle, C. y Sarstedt, M. (2014). A primer on partial least squares structural equation modeling. Recuperado de https://www.researchgate.net/profi le/Qais_Almaamari3/post/How_ca n_I_justify_in_a_reflective_secondorder_construct_to_drop_some_dim ensions_which_not_perform_well/a ttachment/5a79c437b53d2f0bba504 2d9/AS\%3A591053426008064\%40 1517929526701/download/3b.+Hai r+Bo 
Hair, J. F., Hult, G. T., Ringle, C. M., Sarstedt, M., Castillo Apraiz, J., Cepeda Carrión, G. y Roldan, J. L. (2019). Manual de partial least squares structural equation modeling. Recuperado de https://doi.org/10.3926/oss.37

Haverila, M. y Barkhi, R. (2009). The influence of experience, ability and interest on eLearning effectiveness. European Journal of Open, distance and E-learning. Recuperado de https://www.eurodl.org/materials/ contrib/2009/Haverila_Barkhi.htm

Hernández, R., Fernández, C. y Baptista, M. (2014). Metodología de la Investigación. (6ta ed.), México: McGraw-Hill

Henseler, J., Ringle, C. M. y Sarstedt, M. (2015). A new criterion for assessing discriminant validity in variance-based structural equation modeling. Journal of the Academy of Marketing Science. Recuperado de https://doi.org/10.1007/s11747014-0403-8

Hew, K. F. y Cheung, W. S. (2014). Students' and instructors' use of massive open online courses (MOOCs): Motivations and challenges. Educational Research Review. Recuperado de https://doi.org/10.1016/j.edurev.20 14.05.001

Hock, C., Ringle, C. M. y Sarstedt, M. (2010). Management of multipurpose stadiums: Importance and performance measurement of service interfaces. International Journal of Services Technology and Management. Recuperado de https://doi.org/10.1504/IJSTM.20 10.034327

Holmes, B. y Gardner, J. (2006). Elearning: Concepts and practice. United States: SAGE Publications.
Recuperado de http://dx.doi.org/ 10.4135/9781446212585

Hong, S., Thong, J. Y. L. y Tam, K. Y. (2006). Understanding continued information technology usage behavior: A comparison of three models in the context of mobile internet. Decision Support Systems. Recuperado de http://dx.doi.org/ 10.1016/j.dss.2006.03.009

Hsu, M. H., Yen, C. H., Chiu, C. M. y Chang, C. M (2006). A longitudinal investigation of continued online shopping behavior: An extension of the theory of planned behavior. International Journal HumanComputer Studies. Recuperado de http://dx.doi.org/10.1016/j.ijhcs.20 06.04.004

Issa, I. y Hamm U. (2017). Adoption of Organic Farming as an Opportunity for Syrian Farmers of Fresh Fruit and Vegetables: An Application of the Theory of Planned Behaviour and Structural Equation Modelling. Recuperado de https://doi.org/10.3390/su9112024 Kim, Gi., Shin, Bongsik y Lee, Ho. (2009). Understanding dynamics between initial trust and usage intentions of mobile banking. Information System Journal. 19. 283311. Recuperado de https://doi. org/10.1111/j.1365-2575.2007.00

269.x

Koufaris, M. (2002). Applying the technology acceptance model and flow theory to online consumer behavior. Information systems research. Recuperado de https:// doi.org/10.1287/isre.13.2.205.8

Leonard, M., Graham, S. y Bonacum, D. (2004). The human factor: the critical importance of effective teamwork and communication in providing safe care. BMJ Quality y 
Safety. Recuperado de http://dx.doi.org/10.1136/qshc.200 4.010033

Liaw, S. S., Huang, H. M. y Chen, G. D. (2007). Surveying instructor and learner attitudes toward e-learning. Computers \& Education. Recuperado de https://doi.org /10.1016/j.compedu.2006.01.001

Liu, Y. y Chen, N. S. (2008). An adoption model for mobile learning. Proceeding for the International Conference E-Commerce. Recuperado de http://www.iadis portal.org/digital-

library/mdownload /an-adoptionmodel-for-mobile-learning

Luarn, P. y Lin, H.H. (2005) Toward an Understanding of the Behavioral Intention to Use Mobile Banking. Computers in Human Behavior, 21, 873-891. Recuperado de http://dx.doi.org/10.1016/j.chb.20 04.03.003

Ma, J., Hipel, K. W., Hanson, M. L., Cai, X. y Liu, Y. (2018). An analysis of influencing factors on municipal solid waste source-separated collection behavior in Guilin, China by Using the Theory of Planned Behavior. Recuperado de https://doi.org/10.1016/j.scs.2017. 11.037

Maldonado, U. P. T., Khan, G. F., Moon, J. y Rho, J. J. (2011). Elearning motivation and educational portal acceptance in developing countries. Online Information Review. Recuperado de https://doi .org/10.1108/14684521111113597

Malhotra, N. (2008). Investigación de mercados (5ta ed.). México: Pearson Prentice Hall

Melo, A., Ayaviri-Panozo, A., y Rocha, M. (2018). Adaptation of the Curriculum to the Entrepreneurial
Intention: A Study through the Analysis of the PerformanceImportance Map (IPMA). 10th International Symposium on Project Approaches in Engineering Education. Recuperado de https://www.researchgate.net/profi le/Alberto_Ayaviri-

Panozo/publication/323543267_Ad aptation_of_the_Curriculum_to_the _Entrepreneurial_Intention_A_Stud y_through_the_Analysis_of_the_Per formance-

Importance_Map_IPMA/links/5a9 b5a03a6fdcc3cbacb5e8f/Adaptation -of-the-Curriculum-to-the-

Entrepreneurial-Intention-A-Studythrough-the-Analysis-of-thePerformance-Importance-MapIPMA.pdf?origin=publication_detail Mouakket, S. y Bettayeb, A. M. (2015). Investigating the factors influencing continuance usage intention of Learning management systems by university instructors. International Journal of Web Information Systems. Recuperado de https://doi .org/10.1108/IJWIS-03-2015-0008

Moore G.C. y Benbasat I. (1996) Integrating Diffusion of Innovations and Theory of Reasoned Action models to predict utilization of information technology by endusers. Recuperado de https://doi. org/10.1007/978-0-387-34982-4_10

Musa, P. F. (2006). Making a case for modifying the technology acceptance model to account for limited accessibility in developing countries. Information technology for development. Recuperado de https://doi.org/10.1002/itdj.20043

Nunnally, J. y Bernstein, I. (1994). Psychometric theory. Recuperado de https://doi.org/10.1177/ 014662169501900308 
Obringer Lee, Ann (2002). How Elearning Works. Recuperado de http://www.howstuffworks.com/el earning1.html

Oye, D., A. Iahad. y Ab. Rahim, N. Z. (2012). The Impact of UTAUT Model and ICT Theoretical Framework on University Academic Staff: Focus on Adamawa State University, Nigeria. International Journal of Computers \& Technology. Recuperado de https://doi.org/10.24297/ijct.v2i2b. 2640

Parasuraman, A. (2000). Technology Readiness Index (TRI) a multipleitem scale to measure readiness to embrace new technologies. Recuperado de https://doi.org /10.1177/109467050024001

Park S. (2009). "An Analysis of the Technology Acceptance Model in Understanding University Students' Behavioural Intention to Use eLearning". Education Technology \& Society. Recuperado de https://www.jstor.org/stable/jeduct echsoci.12.3.150

Pattansheti, M., Kamble, S. S., Dhume, S. M. y Raut, R. D. (2016). Development, measurement and validation of an integrated technology readiness acceptance and planned behaviour model for Indian mobile banking industry. Recuperado de https://doi.org/ 10.1504/IJBIS.2016.076875

Quezada, C. (2007). Potencia estadística, sensibilidad y tamaño de efecto: ¿un nuevo canon para la investigación? Recuperado de http://onomazein.letras.uc.cl/Artic ulos/16/4_Quezada.pdf

Rogers, E. M. (1995). Diffusion of Innovations: modifications of a model for telecommunications.
Recuperado de https://doi.org/ 10.1007/978-3-642-79868-9_2

Roldán, J. L. y Cepeda, G. (2016). Modelos de ecuaciones estructurales basados en la varianza: Partial least squares (PLS) para investigadores en ciencias sociales. Recuperado de https://fatunasam.com/fatdocs/Ex poWilliam2020.pdf

Salloum, S. A. S. (2018). Investigating students' acceptance of E-learning system in Higher Educational Environments in the UAE: Applying the Extended Technology Acceptance Model (TAM). Recuperado de https://www.researchgate.net/profi le/Said_Salloum/publication/32741 8762_Investigating_students\%27_ac ceptance_of_E-

learning_system_in_Higher_Educati onal_Environments_in_the_UAE_A pplying_the_Extended_Technology _Acceptance_Model_TAM/links/5 b8eb1da45851540d1c88879/Investig ating-students-acceptance-of-Elearning-system-in-Higher-

Educational-Environments-in-theUAE-Applying-the-ExtendedTechnology-Acceptance-ModelTAM.pdf

Samaradiwakara, G. D. M. N. y Gunawardena, C. G. (2014). Comparison of existing technology acceptance theories and models to suggest a well improved theory/model. International Technical Sciences Journal. Recuperado de https://enos.it college.ee/ edmund/infhw/seminaritoo/itsj-spec-1-1-3.pdf Santos, D., Veiga, R. y Moura, L. (2010) Teoria do comportamento planejado decomposto: determinantes de utilização do Serviço Mobile-banking. Revista 
Organizações em Contexto. Recuperado de https://www. metodista.br/revistas/revistasims/in dex.php/OC/article/view/2696/26 39

Sharma, S., Hasteer, N., Mishra, S. P. y Van Belle, J. P. (2016, October). Identifying the contextual relationship among the Agile adoption factors through interpretive structural modeling. International Conference on Information Technology. Recuperado de https://doi.org/10.1109/INCITE.2 016.7857596

Szajna, B. (1996). Empirical evaluation of the revised technology acceptance model. Management Science. Recuperado de https://doi.org/ $10.1287 /$ mnsc. 42.1 .85

Tamrakar, A. y Metha, K. (2011). Analysis of Effectiveness of Web based E-Learning. International Journal of Soft Computing and Engineering (IJSCE), 6(7)

Taylor, S. y Todd, P. (1995). Decomposition and crossover effects in the theory of planned behavior: a study of consumer adoption intentions. International Journal of Research in Marketing. Recuperado de https://doi.org/10.1016/01678116(94)00019-K

Thompson, M. K., Moroni, G., Vaneker, T., Fadel, G., Campbell, R. I., Gibson, I. y Martina, F. (2016). Design for Additive Manufacturing: Trends, opportunities, considerations, and constraints. Recuperado de https://doi.org/10.1016/j.cirp.2016 .05 .004
Vallerand, R. J. (1997). Toward a hierarchical model of intrinsic and extrinsic motivation. Advances in experimental social psychology. Recuperado https://doi.org/10.1016/S00652601(08)60019-2

Venkatesh, V. (2000). Determinants of perceived ease of use: Integrating perceived behavioral control, computer anxiety and enjoyment into the technology acceptance model. Information systems research. Recuperado de https://doi.org/10.1287/isre.11.4.3 42.11872

Venkatesh, V. y Davis, F. D. (2000). A theoretical extension of the technology acceptance model: four longitudinal field studies. Management Science. Recuperado de https://doi.org/10.1287/ mnsc.46.2.186.11926

Venkatesh, V., Thong, J. Y., y Xu, X. (2012). Consumer acceptance and use of information technology: extending the unified theory of acceptance and use of technology. Recuperado de https://doi.org/ 10.2307/41410412

Wagner, E. D. (2005). Enabling mobile learning. Recuperado de https://www.learntechlib.org/p/99 $141 /$

Xie, Q., Song, W., Peng, X. y Shabbir, M. (2017). Predictors for egovernment adoption: integrating TAM, TPB, trust and perceived risk. Recuperado de https://doi.org/ 10.1108/EL-08-2015-0141 